Communications in Physics, Vol. 28, No. 3 (2018), pp. 255-263

DOI:10.15625/0868-3166/28/3/12673

\title{
A THEORETICAL STUDY OF DEFLECTION OF AFM BIMATERIAL CANTILEVERS VERSUS IRRADIATED POSITION
}

\author{
LE TRI DAT ${ }^{a}$, HO THANH HUY ${ }^{a}$ AND NGUYEN DUY VY ${ }^{b, c, \dagger}$ \\ ${ }^{a}$ Faculty of Physics and Engineering Physics, \\ University of Science, Vietnam National University, Ho Chi Minh City, Vietnam \\ ${ }^{b}$ Theoretical Physics Research Group, \\ Advanced Institute of Materials Science, Ton Duc Thang University, Ho Chi Minh City, Vietnam \\ ${ }^{c}$ Faculty of Applied Sciences, Ton Duc Thang University, Ho Chi Minh City, Vietnam \\ ${ }^{\dagger}$ E-mail: nguyenduyvy@tdtu.edu.vn
}

Received 14 June 2018

Accepted for publication 24 August 2018

Published 31 August 2018

\begin{abstract}
The bimaterial cantilevers of atomic force microscopes have been widely used in chemical and bio-sensing. Due to the difference in the thermal expansion coefficients of the two layers, the cantilever is deflected and its deflections is dependent on the heat absorption from the ambient environment or the objects adsorbed on the cantilever surface. In this study, we theoretically examine the deflection of this cantilever considering different irradiated configurations of a laser beam and thicknesses of the coating layer. We show that the temperature difference between the end and the clamped position is maximized for an irradiation at the cantilever end and this difference reduces with increasing coating thickness. Especially, the maximal deflection is seen for an irradiation in the middle of the cantilever, around 0.6 of the cantilever length from the clamped position. The obtained results could help determining an irradiated configuration of laser and the coating thickness to optimize the sensitivity of the cantilevers in thermally sensing devices.
\end{abstract}

Keywords: AFM, bimaterial cantilever, thermal expansion, laser irradiation.

Classification numbers: 07.79.Lh, 78.20.N-, 65.40.De.

(C)2018 Vietnam Academy of Science and Technology 


\section{INTRODUCTION}

The atomic force microscope (AFM), which is used for atomic and nano-scale measurement, has many applications in polymer chemistry and molecular engineering [1-5] and recently in pharmaceutical science [6-8]. When a cantilever scans over a surface of sample, the tip-sample interaction could alter the cantilever deflection and the resonance frequency. Recently, bimaterial cantilevers have been used in chemical and biological measurements where the interaction or adsorption of molecules to the cantilever surface could also deflect and or change the cantilever frequency [8]. By analyzing the response of the cantilever, the properties of the surface could be revealed. Usually, the cantilever deflection is detected by the optical lever detection scheme $[9,10]$ where a laser beam is irradiated on the cantilever and then the reflected laser is absorbed and analyzed by a photodetector.

In recent studies, the cantilever deflection could be effectively controlled if the resonance effect is available where the optical power exerting on the cantilever is strongly enhanced in an optical microcavity $[11,12]$. In this study, we theoretically examine the thermally induced effects on a bimaterial cantilever assuming that the absorbed position is in the middle or at the end of the cantilever, i.e., different laser spot positions $\left(x_{0}\right)$ along the cantilever length $(L)$. In fact, the laser beam also exerts a radiation pressure on the cantilever. This pressure is from the momentum of the photons that imping on the cantilever surface. However, it has been shown that for a direct irradiation, the deflection from the radiation pressure is much less than that from the thermal effect [12]. Therefore, we could examine only the role of the thermally induced deflection in this study.

The cantilever is a rectangular silicon beam (width $w$ and thickness $t_{2}$ ) coated by a gold thin film of thickness $t_{1}$. Thermally induced effects change the cantilever deflection due to the nonhomogeneous thermal expansions of the layers in the cantilever structure. This study will reveal the dependence of the deflection on the coating thickness and on the the irradiated position of the laser. The heat is greatest at the irradiated position and then dissipates to the environment and transfers along the beam (aligned in the $x$ axis). At the cantilever end, $x=L$, the heat flow meets a boundary and is reflected. The clamped position, $x=0$, on the other hand, diminishes all heat transferred from the spot position and keeps the temperature constant, $T(0)=T_{0}$. The metallic thin film has a higher thermal absorption and, therefore, a higher temperature than the Si layer. Furthermore, the Au layer has a higher expansion coefficient. As a result, the cantilever deflects toward the Si layer.

In a recent study, Toda et al. [13], has rederived the analytical formular for the deflection of these bimaterial cantilevers using the theory of Barnes et al. [14]. The calculation has then been confirmed using the Au-SiN $x$ cantilevers thermally excited in vacuum and air. They used an irradiated laser beam at the free end of the cantilever as a heat source. Nevertheless, the heat source at an arbitrary position in the middle of the cantilever for a wider sensing application has not been taken into account.

In this study, we will theoretically examine the case that an assumed heat source locates in the middle of the cantilever. This research calculates the deflection $(z(L))$ under the irradiated configurations (presented via the spot position $x_{0}$ ) and the coating thickness to find the optimal irradiated position for $z(L)$. The local excitation of a soft cantilever could change the amplitude of higher order modes to apply in the biological measurement $[15,16]$. 


\section{AXIAL TEMPERATURE DISTRIBUTION}

The 1-dimensional temperature distribution, $T(x)$, of the cantilever will be derived. For wide cantilevers, the temperature distribution along the width should be taken into account and a complex distribution could be obtained [17]. Nevertheless, the current study assumes a homogeneous temperature in the cantilever cross section.

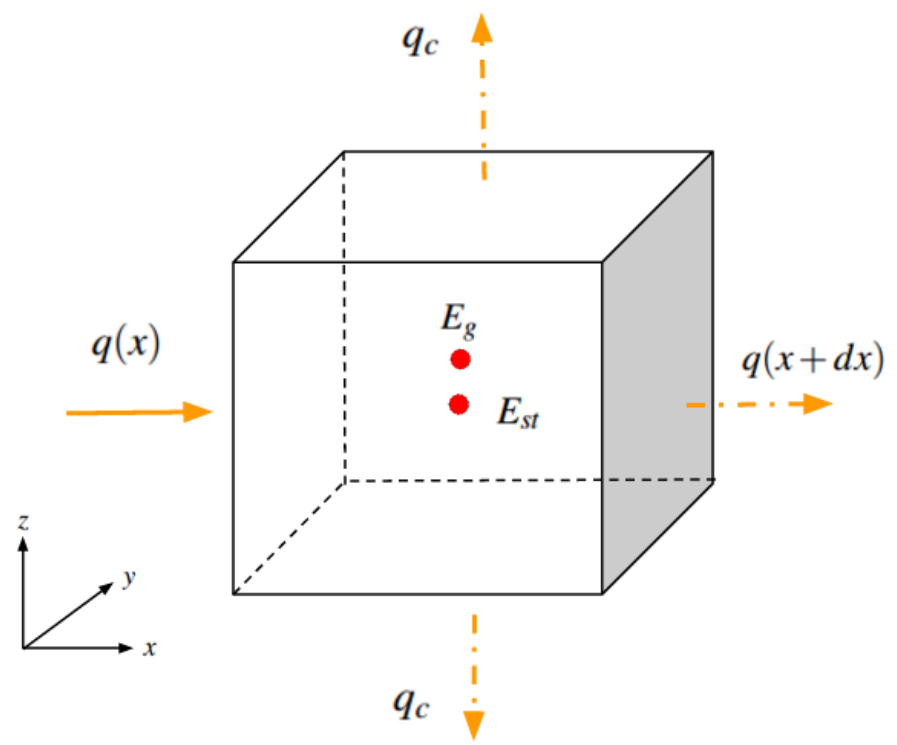

Fig. 1. The model to calculate the temperature distribution along the cantilever. The volume element $d x d y d z$ has an internal generated heat $E_{g}$.

To find the temperature transfer equation, we examine an infinitesimal element [see Fig. 1] on the cantilever axis (the $x$ axis) and apply the principle of energy conservation, i.e., the change in the stored energy, $E_{s t}$, during a time interval $d t$ is equal to the subtraction of the energy entering in, $E_{i n}$, and that leaving out, $E_{\text {out }}$, adding the energy generated, $E_{g}$, in that element.

$$
\frac{d E_{s t}}{d t}=E_{\text {in }}-E_{\text {out }}+E_{g}
$$

We consider only the steady-state conduction, then the temporal component is diminished. The heat transfer rate in the $x$-direction per unit area in the cross section in the material $i$ is $(i=1,2)$

$$
q_{i}=-k_{i} \frac{d T}{d x}
$$

where $k_{i}$ is the thermal conductivity. Furthermore, the convection heat transfer of the top and bottom surfaces is

$$
q_{c}=2 \bar{h}\left[T(x)-T_{0}\right]
$$

Here, $\bar{h}$ is the convection heat transfer coefficient and $T_{0}$ is the temperature at the clamped position (which is also the ambient temperature). Then, one obtains, 


$$
q_{1}(x) A_{1}+q_{2}(x) A_{2}-q_{1}(x+d x) A_{1}-q_{2}(x+d x) A_{2}-q_{c} w d x+g\left(A_{1}+A_{2}\right) d x=0 .
$$

Using the Taylor expansion, $q(x+d x) \simeq q(x)+q^{\prime}(x) d x$, one obtains the temperature distribution equation. That is, from Eq. (4)

$$
\begin{aligned}
& -q_{1}^{\prime}(x) A_{1} d x-q_{2}^{\prime}(x) A_{2} d x-q_{c} w d x+g\left(A_{1}+A_{2}\right) d x=0 \\
\Leftrightarrow & -\left(q_{1}^{\prime}(x) t_{1}+q_{2}^{\prime}(x) t_{2}\right) w d x-q_{c} w d x+g\left(t_{1}+t_{2}\right) w d x=0 \\
\Leftrightarrow & \left(k_{1} t_{1}+k_{2} t_{2}\right) \frac{d^{2} T}{d x^{2}}-2 \bar{h}\left[T(x)-T_{0}\right]+g\left(t_{1}+t_{2}\right)=0 \\
\Leftrightarrow & \frac{d^{2} T}{d x^{2}}-m^{2}\left[T(x)-T_{0}\right]+G=0,
\end{aligned}
$$

where $m^{2}=\frac{2 \bar{h}}{k_{1} t_{1}+k_{2} t_{2}}$ and $g$ is the generated energy rate in the volumetric element. The generated energy is the absorptive power when a laser beam is irradiated on the surface, i.e

$$
\begin{aligned}
E_{g} & =P_{a b s} \Leftrightarrow g d V=\alpha I(x) d S, \\
\Rightarrow g & =\alpha I(x) \frac{d S}{d V}=\frac{\alpha I(x)}{t_{1}+t_{2}}
\end{aligned}
$$

where $\alpha$ is the absorptance coefficient and $I(x)$ is the intensity of laser. The intensity of laser obeys the Gauss distribution, but the beam waist is assumed to close to 0 and has the delta-function form. Then, the G quantity in Eq. (5) has the form as follow

$$
G=\frac{\alpha P}{\left(k_{1} t_{1}+k_{2} t_{2}\right) w \cdot \operatorname{erf}(\sqrt{2})} \delta\left(x-x_{0}\right),
$$

where $\delta\left(x-x_{0}\right)$ is the Delta-Dirac function and $P$ is the input power. Using the Green function method (see Appendix), the solution is

$$
\Delta T(x)=-\frac{\alpha P}{\left(k_{1} t_{1}+k_{2} t_{2}\right) w \operatorname{erf}(\sqrt{2})} \begin{cases}B \sinh m x & \text { for } x<x_{0}, \\ C \cosh m x+D \sinh m x & \text { for } x>x_{0} .\end{cases}
$$

In Fig. 2, the temperature distribution for various laser spot positions, $x_{0} / L=0.2,0.4,0.6$, and 0.8 , are shown. It is seen that the further the irradiated position from the clamped position locates, the higher the temperature peak is obtained. It is of $0.72 \mathrm{~K}$ for $x_{0}=0.8 \mathrm{~L}$ [Fig. 2d), solid line] in comparison to $0.28 \mathrm{~K}$ for $x_{0}=0.2 L$ [Fig. 2a), solid line]. Furthermore, the thinner the coating layer is, the higher the temperature reaches. This is due to the weak dissipation of the absorbed heat of thin films in comparison to that of thicker films. As a result, the greater film thicknesses, e.g., $100 \mathrm{~nm}$ (dotted lines) and $140 \mathrm{~nm}$ (dashed lines) have smaller temperature peaks than the 20 nm- and 60 nm-thick films do. As a result, $T(L)$ is greatest for $x_{0}=L$ as shown in Fig. 3.

The deflection, however, has a different response behavior to the spot position rather than that of the temperature distribution, as shown in the next section and Fig. 4. 

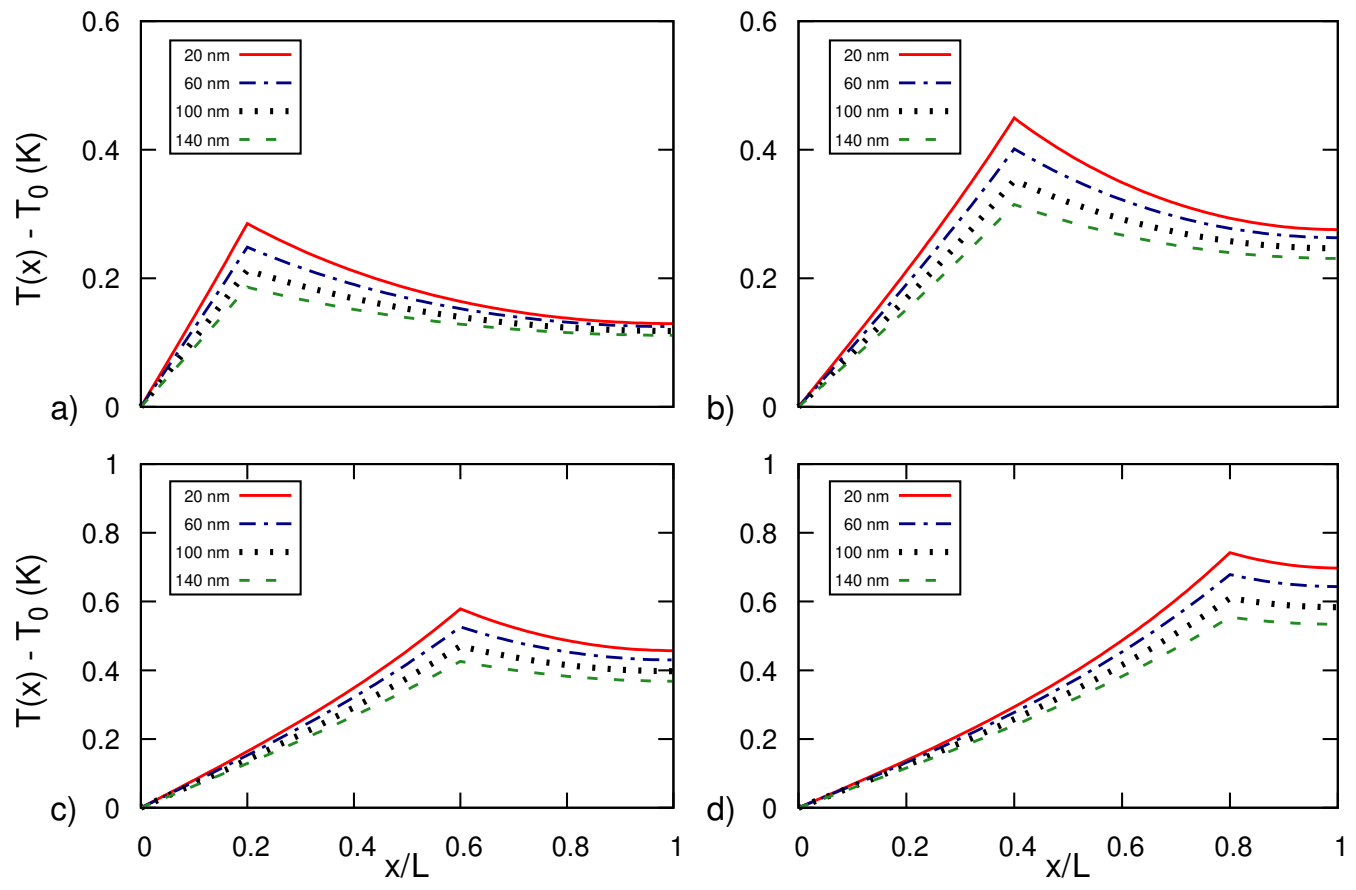

Fig. 2. The temperature distribution when the irradiated position is $x_{0}=0.2 L$ (a), $0.4 L$ (b), $0.6 L(c)$, and $0.8 L(d)$.

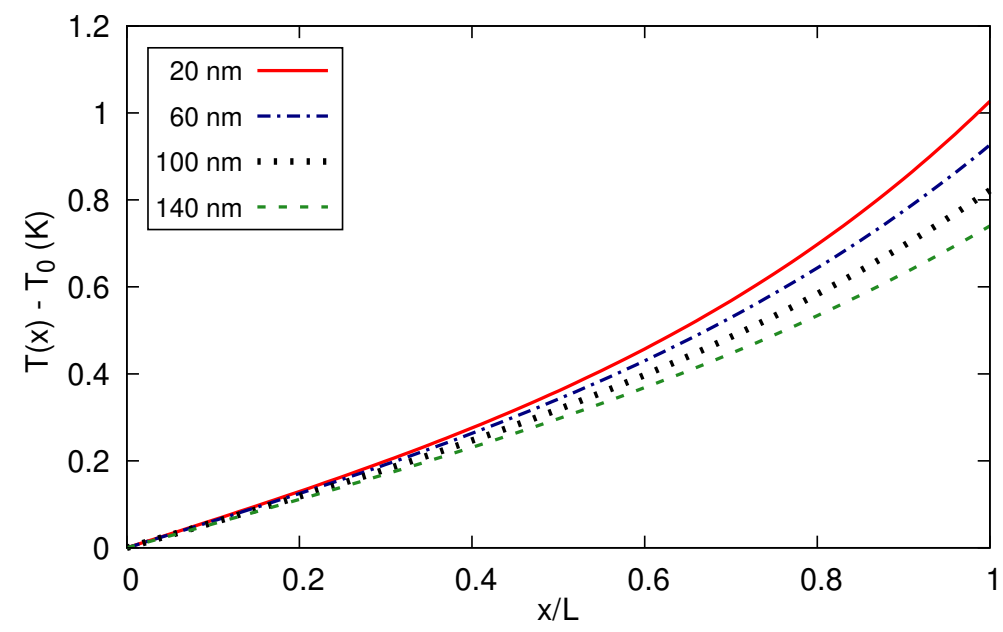

Fig. 3. The temperature distribution when the irradiated position is $x_{0}=L$. 


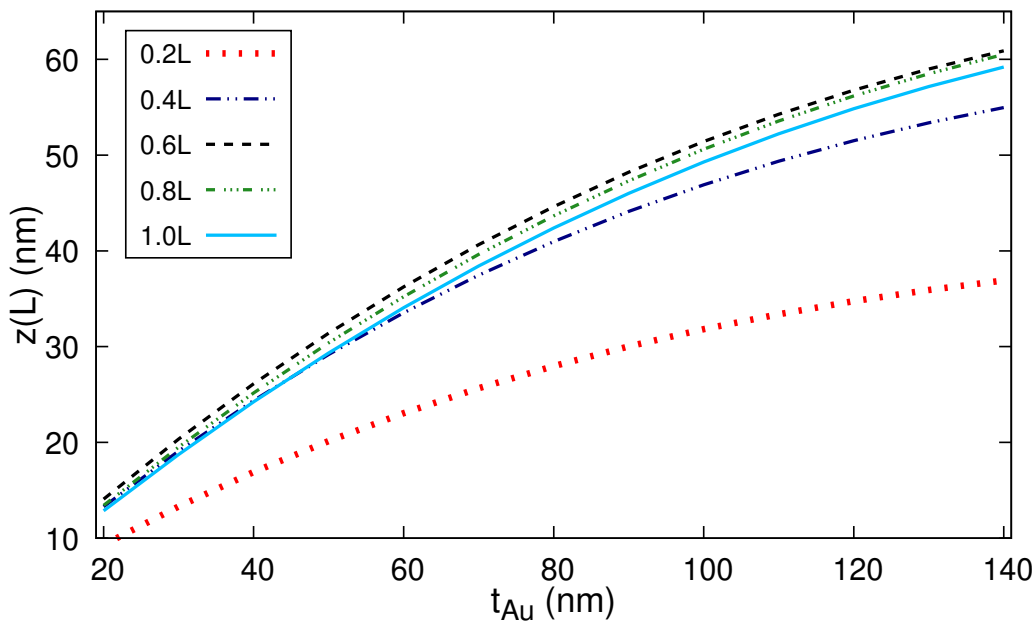

Fig. 4. Cantilever deflection for various Au film thicknesses $t_{A u}=20-140 \mathrm{~nm}$ at the irradiated positions $0.2 L, 0.4 L, 0.6 L, 0.8 L$ and $L$.

\section{CANTILEVER DEFLECTION}

Having the temperature profile, the deflection $z(L)$ could be figured out by using the equation [18],

$$
z^{\prime \prime}(x)=N \Delta T(x)
$$

where

$$
N=\frac{6\left(\gamma_{2}-\gamma_{1}\right)\left(t_{1}+t_{2}\right)}{\left(t_{2}^{2} K\right)}
$$

and

$$
K=4+6\left(\frac{t_{1}}{t_{2}}\right)+4\left(\frac{t_{1}}{t_{2}}\right)^{2}+\frac{E_{1}}{E_{2}}\left(\frac{t_{1}}{t_{2}}\right)^{3}+\left(\frac{E_{2}}{E_{1}}\right)\left(\frac{t_{2}}{t_{1}}\right)
$$

with the parameters showed in Table 1 . To solve this equation, these boundary conditions are followed, $z(0)=0$ and $z^{\prime}(0)=0$. Besides, the continuity conditions are applied, $z^{+}\left(x_{0}\right)=z^{-}\left(x_{0}\right)$ and $z^{\prime+}\left(x_{0}\right)=z^{\prime-}\left(x_{0}\right)$. We obtain the solution of Eq. (10) as follow

$$
z(x)=\frac{\alpha N P}{\left(k_{1} t_{1}+k_{2} t_{2}\right) m w \operatorname{erf}(\sqrt{2})} \begin{cases}B\left[x-\frac{\sinh (m x)}{m}\right] & \text { for } x<x_{0} \\ \xi x+v-\frac{1}{m}[C \cosh (m x)+D \sinh (m x)] & \text { for } x>x_{0}\end{cases}
$$


Table 1. Parameters for calculations.

\begin{tabular}{llll}
\hline Parameters & Symbol (Unit) & Au [1] & Si [2] \\
\hline \hline Thickness & $t(\mathrm{~nm})$ & $20-140$ & 460 \\
Thermal conductivity & $k\left(\mathrm{Wm}^{-1} \mathrm{~K}^{-1}\right)$ & 320 & 100 \\
Young's modulus & $E(\mathrm{GPa})$ & 61.3 & 190.0 \\
Thermal expansion coefficient & $\gamma\left(10^{-6} / K\right)$ & 14.2 & 2.6 \\
\hline
\end{tabular}

Here,

$$
\begin{gathered}
\xi=B\left[1-\cosh \left(m x_{0}\right)\right]+C \sinh \left(m x_{0}\right)+D \cosh \left(m x_{0}\right) \\
v=B\left[x_{0} \cosh \left(m x_{0}\right)-\frac{\sinh \left(m x_{0}\right)}{m}\right]+C\left[\frac{\cosh \left(m x_{0}\right)}{m}-x_{0} \sinh \left(m x_{0}\right)\right] \\
+D\left[\frac{\sinh \left(m x_{0}\right)}{m}-x_{0} \cosh \left(m x_{0}\right)\right] .
\end{gathered}
$$

In Fig. $4, z(L)$ is presented for the irradiated positions of $0.2 L, 0.4 L, 0.6 L, 0.8 L$, and $L$ with the coating thickness of $\mathrm{Au} t_{A u}=20-140 \mathrm{~nm}$. In Fig. 4, a general view of $z(L)$ is shown. We could see that the deflections when $x_{0}=0.6 L$ and $x_{0}=0.8 L$ are greater than that when $x_{0}=0.2 L, x_{0}$ $=0.4 L$ and $x_{0}=L$. This seems to be not consistent with the temperature distribution [see Figs. 2 and 3] where $T(L)$ is greater for smaller $t_{A u}$. However, the deflection $z(L)$ is the accumulated summation of all deflections of every length element along the cantilever, $d z(x)$; therefore, there is a position that could maximize $z(L)$. And in this case, it is $x_{0} \simeq 0.6 L$, as shown in Fig. 4.

\section{CONCLUSIONS}

In this study, we have explored the dependence of an AFM cantilever deflection on the irradiated configuration from a laser source. The temperature distribution for various irradiation positions is analytically presented. The deflection is strongly dependent on the irradiated position, it is greatest for spot position in the middle of the cantilever, around the position of 0.6 of the cantilever length. This value is dependent on the parameters used in the calculation, especially, the mechanical and thermal conductivity of the metallic thin film.

\section{ACKNOWLEDGMENT}

We are thankful to Assoc. Prof. C. M. Hoang (ITIMS) and Prof. L. V. Hoang for encouragement. This research is funded by Vietnam National Foundation for Science and Technology Development (NAFOSTED) under grant number 103.01-2016.70. 


\section{REFERENCES}

[1] N. V. Lavrik, M. J. Sepaniak and P. G. Datskos, Rev. Sci. Instrum. 75 (2004) 2229.

[2] S. Singamaneni, M. LeMieux, H. Lang, C. Gerber, Y. Lam, S. Zauscher, P. Datskos, N. Lavrik, H. Jiang, R. Naik, T. Bunning and V. Tsukruk, Adv. Mater. 20 (2008) 653.

[3] A. Boisen, S. Dohn, S. S. Keller, S. Schmid and M. Tenje, Rep. Prog. Phys. 74 (2011) 036101.

[4] M. F. Khan, N. Miriyala, J. Lee, M. Hassanpourfard, A. Kumar and T. Thundat, Appl. Phys. Lett. 108 (2016) 211906.

[5] G. Zhang, L. Wu, C. Li, S. Wu and Q. Zhang, Rev. Sci. Instrum. 88 (2017) 075007.

[6] W. Taweepreda, S. Tuaybut, S. Puangmanee and T. Khoa, Communications in Physics 24 (2014) 51.

[7] J. Wang, M. J. Morton, C. T. Elliott, N. Karoonuthaisiri, L. Segatori and S. L. Biswal, Anal. Chem. 86 (2014) 1671, PMID: 24417655.

[8] H. Etayash, M. F. Khan, K. Kaur and T. Thundat, Nat. Commun. 7 (2016) 12947.

[9] P. S. Jung and D. R. Yaniv, Scanning force microscope with beam tracking lens, 021994.

[10] T. J. Yang, G. A. Lessard and S. R. Quake, Applied Physics Letters 76 (2000) 378.

[11] N. D. Vy and T. Iida, Applied Physics Letters 102 (2013) 091101.

[12] N. D. Vy and T. Iida, Applied Physics Express 9 (2016) 126601.

[13] M. Toda, T. Ono, F. Liu and I. Voiculescu, Rev. Sci. Instrum. 81 (2010) 055104.

[14] J. R. Barnes, R. J. Stephenson, C. N. Woodburn, S. J. OShea, M. E. Welland, T. Rayment, J. K. Gimzewski and C. Gerber, Rev. Sci. Instrum. 65 (1994) 3793.

[15] C. M. Hoang, N. D. Vy, L. T. Dat and T. Iida, Japanese Journal of Applied Physics 56 (2017) 06 GK05.

[16] C. M. Hoang, T. Iida, L. T. Dat, H. T. Huy and N. D. Vy, Optics Communications 403 (2017) 150.

[17] J. R. Serrano, L. M. Phinney and J. W. Rogers, Int. J. Heat Mass Transfer 52 (2009) 2255.

[18] W. C. Young and R. G. Budynas, Roark's formulas for stress and strain, vol. 7, McGraw-Hill New York, 2002.

\section{APPENDIX}

To solve Eq. (5), the Green function method has been used and the following boundary conditions are applied. At the clamped position, $x=0, T(0)=T_{0}$.

At $x=L$,

$$
-\left(k_{1} t_{1}+k_{2} t_{2}\right) \frac{d T(L)}{d x}=\bar{h}\left(t_{1}+t_{2}\right)\left[T(L)-T_{0}\right] .
$$

The solution of Eq. (5) is

$$
\Delta T(x)=T(x)-T_{0}=-g_{0} g\left(x-x_{0}\right),
$$

and in the following $\Delta T(x)$ is used instead of $T(x)-T(0)$ and $g_{0}=(\alpha P) /\left[\left(k_{1} t_{1}+k_{2} t_{2}\right) w \operatorname{erf}(\sqrt{2})\right]$ for brevity. Here, $g\left(x, x_{0}\right)$ is the Green function which is the solution of the equation

$$
\left(\frac{d^{2}}{d x^{2}}-m^{2}\right) g\left(x, x_{0}\right)=\delta\left(x-x_{0}\right)
$$

Then, one obtains

$$
g\left(x, x_{0}\right)= \begin{cases}A \cosh m x+B \sinh m x & \text { for } x<x_{0}, \\ C \cosh m x+D \sinh m x & \text { for } x>x_{0}\end{cases}
$$


The coefficients $A, B, C$, and $D$ are obtained with the boundary conditions and the continuity of the Green function. $T(0)=T_{0}$ gives $g\left(0, x_{0}\right)=0 \Rightarrow A=0$, and

$$
\begin{aligned}
-\left(k_{1} t_{1}+k_{2} t_{2}\right) \frac{d T(L)}{d x} & =\bar{h}\left(t_{1}+t_{2}\right)\left[T(L)-T_{0}\right] \\
\Rightarrow \frac{d g\left(L, x_{0}\right)}{d x} & =-\frac{\bar{h}\left(t_{1}+t_{2}\right)}{k_{1} t_{1}+k_{2} t_{2}} g\left(L, x_{0}\right) .
\end{aligned}
$$

Therefore,

$$
g\left(x, x_{0}\right)=B \sinh m x,
$$

and,

$$
D=-\frac{\sinh m L+\frac{\bar{h}\left(t_{1}+t_{2}\right)}{m\left(k_{1} t_{1}+k_{2} t_{2}\right)} \cosh m L}{\cosh m L+\frac{\bar{h}\left(t_{1}+t_{2}\right)}{m\left(k_{1} t_{1}+k_{2} t_{2}\right)} \sinh m L} C .
$$

Using the continuity of Green function,

$$
\begin{gathered}
g\left(x_{0}+\epsilon, x_{0}\right)=g\left(x_{0}-\epsilon, x_{0}\right), \\
\frac{d g\left(x_{0}+\epsilon, x_{0}\right)}{d x}-\frac{d g\left(x-\epsilon, x_{0}\right)}{d x}=1,
\end{gathered}
$$

then we have

$$
\begin{aligned}
& C=-\frac{1}{m} \sinh m x_{0} . \\
& B=\frac{\cosh m x_{0}}{\sinh m x_{0}} C+D=-\frac{\cosh m x_{0}}{m}+D .
\end{aligned}
$$

Finally, the temperature distribution are

$$
\Delta T(x)=-g_{0} \begin{cases}B \sinh m x & \text { for } x<x_{0}, \\ C \cosh m x+D \sinh m x & \text { for } x>x_{0} .\end{cases}
$$

